\title{
Opioidgewöhnte Patienten Perioperatives Management
}

Carsten Stoetzer • Andreas Leffler • Jörg Filitz

Im perioperativen Umfeld sind Patienten mit chronischen Schmerzen sowie Patienten mit Suchtanamnese für den klinisch tätigen Anästhesisten häufig eine Herausforderung. Dies gilt im besonderen Maße, wenn eine langfristige Einnahme von Opioiden erkennbar ist. Neben pathophysiologischen Veränderungen bringen diese Patientengruppen häufig logistische Besonderheiten mit sich. Obwohl es sich bei chronischen Schmerzen einerseits bzw. einer Suchterkrankung andererseits grundsätzlich um sehr verschiedene Krankheitsbilder mit z. T. sehr differenten Behandlungsansätzen handelt, weisen sie in der perioperativen Betreuung Parallelen auf, aus denen partiell ein ähnliches Management resultieren kann. Der Artikel befasst sich mit den pathophysiologischen Gegebenheiten bei diesen Patienten, erläutert verschiedene Therapieoptionen und zeigt Gemeinsamkeiten auf.

\section{Einteilung der verschiedenen Patientengruppen}

Ehemals abhängige Patienten Unter dem Begriff der opioidgewöhnten Patienten lassen sich verschiedene Gruppen definieren: Die 1. Gruppe besteht aus Patienten, die ehemals abhängig waren, erfolgreich entwöhnt wurden und keine Substitutionstherapie benötigen. Sie befinden sich in einem stabilen Zustand. $\mathrm{Zu}$ dieser Gruppe zählen auch die Patienten, die über einen relevant langen Zeitraum mit Opioiden behandelt werden mussten, diese aber nicht mehr benötigen.

Patienten unter Substitution Eine weitere Gruppe sind die Patienten, die zwar noch unter einer Suchterkrankung leiden, jedoch stabil substituiert werden. Seit 2002 ist die Zahl der gemeldeten Substitutionspatienten in Deutschland stetig gestiegen: So waren 2013 rund 77000 Patienten beim Bundesinstitut für Arzneimittel und Medizinprodukte (BfArM) mit einer Substitutionstherapie gemeldet [1].

Abhängige Patienten Deutlich komplexer in der perioperativen Betreuung sind die Patienten, die unter einer Abhängigkeit leiden. Der epidemiologische Sucht-Survey von 2012 zeigt, dass
2,3 Mio. Menschen in Deutschland von Medikamenten abhängig sind [2]; 319000 Personen konsumieren Cannabis, Kokain oder Amphetamine [3]. 9,5 Mio. Menschen in Deutschland konsumieren Alkohol in gesundheitsschädlichen Mengen und etwa 1,77 Mio. Menschen gelten als alkoholabhängig [4].

- Als Anästhesisten begegnen wir also entsprechend häufig dem Problem einer Polytoxykomanie.

Patienten mit chronischen Schmerzen Die 4. Gruppe besteht aus Patienten, die seit Jahren unter persistierenden Schmerzen leiden und aufgrund einer hohen Schmerzintensität dauerhaft mit Opioiden therapiert werden: Chronische Schmerzen treten in Deutschland mit einer Prävalenz von $17 \%$, in Europa von 19\% auf [5].

Zusammenfassend lassen sich also folgende Patientengruppen definieren ( $\odot$ Abb. 1):

- ehemalige Abhängige, die keine Substitution mehr benötigen

- Patienten mit bestehender Suchterkrankung, die stabil substituiert werden

- Patienten mit bestehender Suchterkrankung, die sich in keiner Substitutionstherapie befinden

- chronische Schmerzpatienten mit einem hohen Opioidbedarf

\section{Erhöhter Versorgungsaufwand durch pathophysiologische Prozesse}

Voraussetzung Die nachfolgend beschriebenen Phänomene müssen identifiziert werden, um erfolgreich die Therapie anzupassen. Grundsätzlich ist dabei zunächst sicher auszuschließen, dass es sich bei dem verminderten Ansprechen einer etablierten Therapie mit Opioiden ursächlich um einen Krankheitsprogress bzw. die Manifestation neu hinzugetretener Pathologien handelt. 


\section{Toleranz}

$\nabla$

Phänomen Die Toleranz ist ein üblicherweise als Rechtsverschiebung in der Dosis-WirkungsKurve beschriebenes Phänomen. Die Aktivierung exzitatorischer Systeme sowie Rezeptorinternalisierungsprozesse, welche die Anzahl aktivierbarer $\mu$-Opioidrezeptoren verringern, sind an der Entwicklung beteiligt. Dies bedeutet, dass im Verlauf einer Opioidtherapie stetig größere Dosierungen des Opioids benötigt werden, um die erwünschte Schmerzreduktion zu erzielen. Oftmals ist der Wechsel zu einem alternativen Opioid erforderlich.

Entscheidend ist, dass im Rahmen einer Toleranzentwicklung keine Veränderung der ursprünglich zu behandelnden (krankheits- / traumabedingten) Schmerzintensität und keine Herabsetzung der Schmerzempfindungsschwellen für nozizeptive Stimuli stattfinden. Hier kann der Schlüssel für die therapeutische Ausrichtung im Hinblick auf die Abgrenzung zur opioidinduzierten Hyperalgesie liegen [6-11].

\section{Opioidinduzierte Hyperalgesie \\ $\nabla$}

Klinische Relevanz Bis zum gegenwärtigen Zeitpunkt konnte nicht eindeutig geklärt werden, ob und in welchem Ausmaß eine opioidinduzierte Hyperalgesie $(\mathrm{OIH})$ beim Menschen klinische Relevanz besitzt. Ursprünglich wurde davon ausgegangen, dass eine $\mathrm{OIH}$ in erster Linie Ausdruck eines Opioidentzugs ist. In der neueren Literatur finden sich vermehrt Hinweise darauf, dass dieses Phänomen in gleicher Weise unter fortgesetzter Opioidtherapie - möglicherweise beeinflusst von der Dynamik einer Dosissteigerung - auftreten kann [12-14].

Definition Hyperalgesie allgemein ist die klinische Manifestation einer erhöhten neuronalen Aktivität nozizeptiver Bahnen bzw. zentralnervöser Verarbeitungszentren auf Synapsenebene. Sie kann sowohl durch primär erhöhten und ggf. anhaltenden nozizeptiven Input als auch durch Interaktionen endogener oder exogener Substanzen/Medikamente mit prä- oder postsynaptischen Signaltransduktionswegen hervorgerufen werden. Für den Fall der medikamentösen Interaktion von Opioiden scheinen 2 Hauptaktivierungswege eine herausragende Rolle zu spielen:

- zum einen die indirekte Aktivierung postsynaptischer NMDA-Rezeptoren und

- zum anderen die Aktivierung fazilitativer serotonerger Bahnen mit Ursprung im Bereich des Hirnstamms und Projektion nach spinal zu den nozizeptiven Umschaltstellen des Hinterhorns.

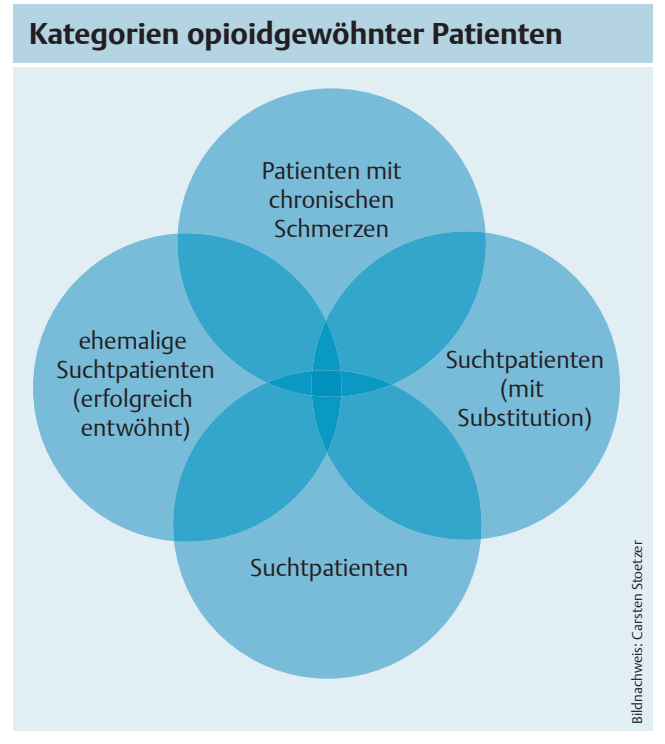

Abb. 1 Opioidgewöhnte Patienten lassen sich in 4 verschiedene Kategorien einteilen. Während die ehemaligen Suchtpatienten mit und ohne Substitution in der Regel kein besonderes Management erfordern, sind Patienten mit chronischen Schmerzen oder aktiver Sucht häufig problematisch.
Die Summe pronozizeptiver Effekte kann von der chemischen Struktur der Opioide mitbestimmt sein $[14,15]$.

Unterschied zur Toleranz Beim Phänomen der Toleranz sind durch eine Rechtsverschiebung der Dosis-Wirkungs-Kurve stets höhere Dosierungen notwendig, um den gewünschten Effekt zu erzielen. Die Schmerzempfindungsschwelle für nozizeptive Stimuli wird jedoch nicht herabgesetzt. Dadurch unterscheidet sie sich von der OIH.

\section{Entzug und Hyperalgesiephänomene}

Symptome Einige Mechanismen, die für eine erhöhte Sensibilität auf nozizeptive Stimuli (Hyperalgesie/OIH) verantwortlich sind, finden sich auch bei Unterbrechung einer Opioidtherapie. Beim Auftreten eines Entzugssyndroms kann daher die Hyperalgesie als klinisches Symptom ebenfalls eine Rolle spielen. Während bei der OIH zeitliche Aspekte weniger im Vordergrund stehen, scheint die Ausprägung ähnlicher Symptome im Rahmen eines Entzugssyndroms deutlicher von Dosisniveau und Anwendungsdauer abhängig zu sein. Für die Ausprägung eines akuten Entzugssyndroms nach singulärer Opioidgabe hat scheinbar v.a. die Kinetik des Opioids eine Bedeutung. Für die Anwendung des kurzwirksamen starken Opioids Remifentanil bei opioidvorbehandelten Patienten sind Entzugssymptome mit Hyperalgesie wiederholt beschrieben worden $[10,16,17]$.

Für die Ausprägung eines akuten Entzugssyndroms nach singulärer Opioidgabe scheint v.a. die Kinetik des Opioids entscheidend zu sein. 


\section{Perioperatives Vorgehen}

\section{Allgemeine Grundlagen}

Narkoseverfahren Grundsätzlich können bei den Patienten mit vorbestehender Opioidtherapie oder aktiver Drogenabhängigkeit alle gängigen Narkoseverfahren angewendet werden. Es empfiehlt sich hierbei, die übliche Vorgehensweise der jeweiligen Klinik fortzuführen.

- Allerdings sollten bei opioidgewöhnten Patienten keine Antagonisten (Naloxon, Flumazenil) verwendet werden.

Auch das sehr kurzwirksame Remifentanil sollte nicht eingesetzt werden, da es einen akuten Entzug induzieren kann [18] ( $\odot$ Tab. 1).

\section{Logistische Besonderheiten}

Patienten mit Substanzmissbrauch Patienten mit einem aktiven Medikamenten- oder Drogenmissbrauch sind als Risikopatienten einzustufen, deren Behandlung ein spezielles anästhesiologisches Management erfordert [19]. Patienten, die über einen Zeitraum > 1 Monat täglich größere Mengen orales Morphin zu sich nehmen, sind gefährdet, eine Opioidtoleranz entwickelt zu haben bzw. zu entwickeln. Sie haben intraoperativ einen 30-100\% höheren Opioidbedarf als opioidnaive Patienten [20]. Da sowohl eine Schmerzexazerbation als auch der Entzug mit ähnlichen Symptomen (Unruhe, Zittern, Kaltschweißigkeit, Zunahme der Schmerzen) verbunden sein kann, ist eine Differenzierung häufig erschwert.

Tab. 2 (unten) Schematische Darstellung des Therapiealgorithmus.
Es empfiehlt sich daher, im Vorfeld diese Patienten zu identifizieren und in einem interdisziplinären Team das Therapiekonzept zu erarbeiten.

Vorgehen an der MHH An der Medizinischen Hochschule Hannover werden die elektiven Patienten in der Prämedikationsambulanz detektiert und dann bereits präoperativ dem Akutschmerzdienst (ASD) vorgestellt. Für Notfälle steht rund um die Uhr ein ärztlicher Mitarbeiter des ASD zur Verfügung.

\section{Prozedurales Management der einzelnen Patientengruppen \\ $\nabla$}

Ehemalige Abhängige ohne Substitution Ein häufiges Problem im Umgang mit ehemaligen Suchtpatienten ist der oft unberechtigt zurückhaltende Einsatz von Opioid-Analgetika aus der Angst heraus, man könne durch die Gabe von Opioiden einen Rückfall induzieren. Obwohl es wichtig ist, das bewusste Erleben der psychotropen Opioidwirkung zu vermeiden, kann eine analgetische Unterversorgung - z.B. durch starke Schmerzen nach großen Operationen und die damit verbundene Stresssituation - die Rückfallgefahr per se erhöhen [21]. Zudem führt eine titrationsweise Applikation von Opioiden in der Behandlung starker Schmerzen nicht zur Induktion psychotroper Nebenwirkungen [22].

Nicht-Opioid-Analgetika Postoperativ werden diese Patienten zunächst mit einem Nicht-Opioid-Analgetikum (NOPA) behandelt. Da sich deren analgetische Wirkung nur begrenzt durch eine Dosiserhöhung steigern lässt ( $\bigcirc$ Tab. 2), sollte bei ausreichend hoher Dosierung eines NOPA und inadäquater Analgesie frühzeitig auf ein Opioid eskaliert werden. Die Kombination von 2 oder mehr NOPA wird kritisch diskutiert, da sie eher zu einer Aggravierung der unerwünschten Arzneimittelwirkung (UAW) bei ausbleibender Steigerung der Analgesie führt [23].

Cave Die Angst vor Opioidnebenwirkungen darf nicht zu einer analgetischen Unterversorgung der Patienten führen, weil so die Rückfallgefahr erhöht wird.

Substitutions-/ bestehende Opioidtherapie Dieser Abschnitt behandelt die Patienten, die entweder aufgrund persistierender Schmerzen mit Opioiden behandelt werden oder ehemals drogenabhängig waren und sich in einer Substitutionstherapie befinden. In Deutschland üblich ist entweder eine Substitution mit Methadon oder dem partialen Opioidrezeptor-Agonisten Buprenorphin $\left(\right.$ Subutex $^{\circledR}$ ). 
Auf den ersten Blick mag es merkwürdig erscheinen, dass in ihrem Wesen so gegensätzliche Krankheitsbilder wie Suchterkrankung und Schmerzkrankheit in einem Patientenkollektiv zusammengefasst werden. Unter dem Aspekt des anästhesiologischen Managements mit besonderer Berücksichtigung der chronischen Opioideinnahme weisen jedoch typischerweise auftretende Spannungsfelder durchaus weitreichende Parallelen auf.

Methadon Es muss zwingend zwischen Levomethadon und Methadonracemat unterschieden werden.

In der perioperativen Phase sollten die Dosierungen des Patienten wie gewohnt beibehalten werden. Hierzu sind die oft zuverlässigen Angaben des Patienten sehr wichtig. Sollte ein Wechsel von Methadonracemat auf Levomethadon notwendig werden, gelten folgende Umrechnungen:

$\checkmark 10 \mathrm{mg}$ Methadonracemat p. 0 .

$=5 \mathrm{mg}$ Levomethadon p.o.

$=2,5 \mathrm{mg}$ Levomethadon s. $\mathrm{c}$

Buprenorphin Im Umgang mit Buprenorphin als nahezu einzigem Vertreter von Partialagonisten mit $\mu$-Opioidrezeptor-Agonismus und $\mathrm{k}$ Opioidrezeptor-Antagonismus im klinischen Alltag besteht häufig große Unsicherheit darüber, inwieweit unerwünschte Interaktionen mit reinen $\mu$-Agonisten klinische Relevanz erlangen oder sogar zu klinisch kritischen Situationen wie akutem Entzugssyndrom führen können.

- Klinische Studien an gesunden Probanden haben jedoch - ebenso wie die klinische Praxis der letzten 20 Jahre - gezeigt, dass eine Vorbehandlung mit z.B. transdermalen Buprenorphin-Systemen die Anwendung von $\mu$-Agonisten wie Fentanyl, Sufentanil oder Piritramid nicht negativ beeinflusst, sondern stets synergistische bzw. additive Effekte erkennen lässt [24].

Auch die im Ausnahmefall durchaus anwendbare Alternative der sublingual-transmukosalen Gabe von Buprenorphin bei Vorbehandlung mit reinen $\mu$-Agonisten (z.B. Fentanyl-TTS) führt im Regelfall zu keinen nachteiligen Interaktionen. Einzige Ausnahme sind mit Methadon oder Levomethadon substituierte Suchtpatienten: Hier gilt weiterhin die strikte Regel der ausschließlichen Kombination reiner $\mu$-Agonisten.

Dosierung von Buprenorphin Wird Buprenorphin zur Substitution eingesetzt, sind oft Tagesdosen von $2-8 \mathrm{mg}$ notwendig. Bei diesen hohen Dosierungen besteht die Gefahr einer Wirkbehinderung reiner $\mu$-OpioidrezeptorAgonisten [25]. Daher empfiehlt sich bei Patienten mit diesen hohen Dosierungen eine präoperative Umstellung auf reine $\mu$-OpioidrezeptorAgonisten, wenn eine große Operation mit hohem Schmerzaufkommen erwartet wird. Obwohl in der Literatur eine Umstellung auf das langwirksame Methadon empfohlen wird [26], verwenden wir in der medizinischen Hochschule Hannover für eine perioperative Umstellung das Hydromorphon (Palladon ${ }^{\circledR}$ ), da es bei pharmakologisch geringem Interaktionspotenzial eine gute Steuerbarkeit aufzeigt.

Wichtig ist, die zuvor bestehende Substitutionstherapie zur gewohnten Zeit beizubehalten. Die vorbestehende Therapie dient jedoch nur als Entzugsprophylaxe. Die Therapie muss daher zwingend erweitert werden, um den operativen Schmerz mit zu behandeln.

Für alle Patientengruppen gilt: Sollte für den Eingriff ein regionalanästhesiologisches Verfahren alleine oder eine Kombination aus Regional- und Allgemeinanästhesie infrage kommen, sollte auch ein Regionalanästhesieverfahren angewendet werden [27].

Partialagonisten wie das Buprenorphin können problemlos mit reinen $\mu$-Agonisten kombiniert werden, ohne dass eine Wirkminderung eintritt. Bei der Vorbehandlung mit (Levo-)Methadon sollten jedoch keine Partialagonisten Verwendung finden.

Umgang mit transdermalen Systemen Für Patienten, die präoperativ mit einem transdermalen System behandelt werden, bestimmt das Ausmaß der Operation das Vorgehen: Bei kleinen und mittleren Operationen, bei denen mit einer intakten Hautdurchblutung und einer regelhaften Resorption des Wirkstoffs zu rechnen ist, kann das Pflaster belassen und postoperativ mit einer für das Ausmaß der Operation angemessenen Schmerztherapie erweitert werden (Pflaster = Basismedikation; ○Tab. 2) [27]. Bei größeren Operationen empfiehlt es sich, das Pflaster nach der Einleitung zu entfernen und durch die i.v. Gabe eines reinen $\mu$-Agonisten $\mathrm{zu}$ ersetzen (० Abb. 2) [28].

Abb. 2 Daten aus [28].
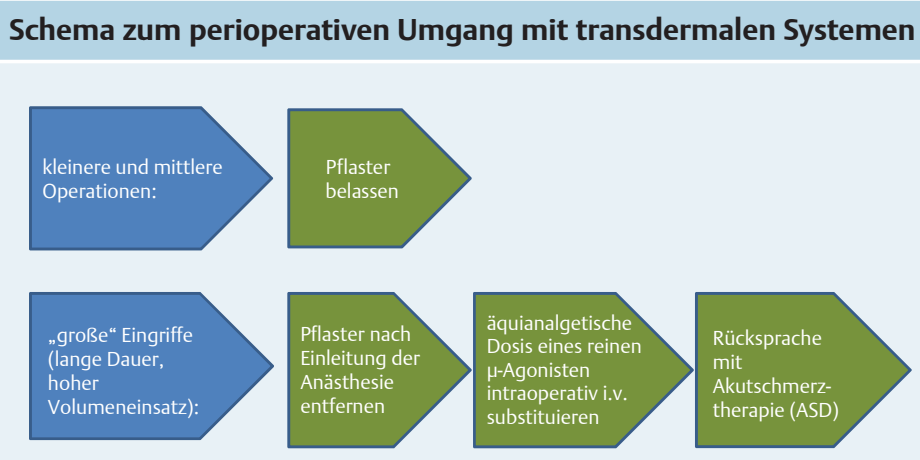

Hypothermie oder Hypovolämie, Katecholamine führen zu verminderter Hautdurchblutung! 
Abb. 3 Allgemeiner

Patienten mit bestehender Sucht ohne Substitution Eine besondere Herausforderung für den Anästhesisten sind die Patienten, die aktuell nicht an einer langfristigen Substitutionstherapie teilnehmen oder diese abgebrochen haben.

- Bei diesen Patienten besteht perioperativ ein hohes Risiko, ein Entzugssyndrom zu entwickeln.

Kommen diese Patienten unter dem Einfluss psychotroper Substanzen wie Alkohol oder anderer Drogen in die Klinik, ist eine Anästhesie mit Ausnahme vital bedrohlicher Indikationen zunächst kontraindiziert [29]. Einige Autoren empfehlen bei diesen Patienten eine perioperative Einstellung auf Methadon zur Prophylaxe von Entzugssymptomen [28]. Auch für diese Patienten konnten wir in unserer Klinik eine suffiziente Stabilisierung bei guter Steuerbarkeit durch Hydromorphon erreichen. Diese kurze perioperative Substitution hilft, die Situation des Patienten zu stabilisieren und schafft Vertrauen.
Dadurch steigt wieder die Compliance und die Gefahr sinkt, dass ein unkontrollierter Beigebrauch anderer psychotroper Substanzen praktiziert wird.

\section{Ambulante Operationen}

Vergesellschaftete Probleme Obwohl heutzutage eine große Zahl operativer Eingriffe in der Regel problemlos ambulant durchführbar ist, kann die Gruppe der Patienten mit bestehender Opioidtherapie ebenso wie die Gruppe der abhängigen Patienten im ambulanten Bereich mit besonderen Problemen vergesellschaftet sein. Daher empfiehlt es sich, auch kleinere Operationen - mit an sich geringem anästhesiologischen Risiko - bei diesen speziellen Patienten in einem stationären Umfeld $\mathrm{zu}$ behandeln und einen Krankenhausaufenthalt für einige Tage zu planen.

Algorithmus.

Perioperative Therapie opioidgewöhnter Patienten

Verabreichung der täglichen Dosis in gewohnter Menge, zur gewohnten Zeit oder: äquianalgetisch i.v. Substitution ( $\mu$-Agonist)
Patienten mit chronischen Schmerzen

- Tumorschmerz

- nicht tumorbedintger

chronischer Schmerz

Verabreichung der täglichen Dosis in gewohnter Menge, zur gewohnten Zeit

perioperativ reiner $\mu$-Agonist (Levomethadon, Morphin, Piritramid, Hydromorphon etc.) über einige Tage; ggf. ergänzend Clonidin

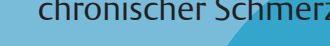

Analgetische Unterversorgung erhöht Rückfallrisiko! Opioide sind erlaubt 
Opioidgewöhnte Patienten bedingen einen erhöhten logistischen Aufwand: In der Regel werden deutlich erhöhte Opioiddosen für eine adäquate Analgesie benötigt. Der zwischenmenschliche Umgang wird durch eine Wechselwirkung zwischen psychischen und organischen Einflüssen erschwert ( $\odot$ Abb. 3 ).

\section{Koanalgetika und Adjuvanzien}

\section{Pregabalin und Gabapentin \\ $\nabla$}

Mittel der Wahl Pregabalin und Gabapentin sind gemäß aktueller Leitlinie Mittel der ersten Wahl für die Therapie neuropathischer Schmerzen. Zur Verwendung der beiden Medikamente für die perioperative Schmerztherapie - präund/oder postoperativ gegeben - liegen mittlerweile weit über 100 klinische Studien vor. Diese kommen fast ausnahmslos zu dem Ergebnis, dass beide Substanzen die Intensität postoperativer Schmerzen und / oder den postoperativen Opioidbedarf reduzieren [30-34]. Daher könnte der präund/oder postoperative Einsatz von Pregabalin oder Gabapentin auch bei chronischen Schmerzpatienten oder bei Patienten mit bestehender Drogenabhängigkeit einen positiven Effekt haben. Allerdings fehlen hier noch weitere Studien, um eine generelle Empfehlung geben zu können.

Missbräuchliche Anwendung Seit 2008 werden für Pregabalin zudem vermehrt missbräuchliche Anwendungen bis hin zu manifestem Suchtverhalten berichtet. Eine 2012 veröffentlichte Analyse der verfügbaren Daten ergab, dass v.a. männliche Patienten mittleren Alters mit früheren polytoxikomanen Episoden für die missbräuchliche Verwendung von Pregabalin prädisponiert zu sein scheinen [35]. Da Pregabalin gegenwärtig in verstärktem Maße zur ambulanten Behandlung neuropathischer und allgemein chronischer Schmerzen mit stetiger Erweiterung des Indikationsspektrums angewendet wird, ist hier ein relevantes Risikopotenzial zu berücksichtigen. Entsprechende Zurückhaltung bei langfristiger Behandlung ist bei Patienten mit Suchtanamnese anzuraten.

\footnotetext{
Die analgetische Wirksamkeit von Pregabalin bei operativen Schmerzen konnte in vielen Studien bewiesen werden. Daher könnte es auch in der Behandlung opioidgewöhnter Patienten eine Rolle spielen. Klinische Studien zum Einsatz von Pregabalin oder Gabapentin bei opioidgewöhnten Patienten existieren noch nicht. Aufgrund des Missbrauchpotenzials ist jedoch bei langfristiger Behandlung von Patienten mit Suchtanamnese ein zurückhaltender Einsatz anzuraten.
}

\section{Dexmedetomidin und Clonidin \\ $\nabla$}

Wirkungsweise Diese Substanzen wirken als potente $\alpha 2$-Agonisten wahrscheinlich durch die Aktivierung des deszendierenden noradrenergen Systems antinozizeptiv. Dexmedetomidin ist chemisch dem Clonidin verwand, verglichen mit diesem etwa 8-mal spezifischer in der Wirkung auf $\alpha 2$-Rezeptoren. Dadurch ist es effektiver in der Sedierung und Analgesie als Clonidin, zeigt aber weniger kardiovaskuläre Nebenwirkungen [36].

Studienlage Eine Metaanalyse von 30 randomisierten klinischen Studien zur perioperativen Gabe von Dexmedetomidin oder Clonidin zeigte, dass beide Substanzen den postoperativen Opioidverbrauch sowie die Schmerzintensität innerhalb der ersten $24 \mathrm{~h}$ signifikant reduzieren [37]. Zusätzlich verringerten beide Substanzen die postoperative Übelkeit, allerdings sind dabei Hypotonie (Clonidin) und Bradykardie (Dexmedetomidin) relevante Nebenwirkungen. Eine neuere Metaanalyse zum Einsatz von Dexmedetomidin zur perioperativen Schmerztherapie kam zu fast identischen Ergebnissen, d.h. zeigte eine Reduktion des Schmerzes und des Opioidverbrauchs sowie ein geringeres Risiko für postoperative Übelkeit [38].

- Die aktuelle Datenlage lässt demnach vermuten, dass Dexmedetomidin und Clonidin auch bei opioidgewöhnten Patienten oder bei Patienten mit einem bestehenden Drogenabusus günstige Effekte auf das postoperative Schmerzniveau haben könnten.

Die $\alpha 2$-Agonisten Dexmedetomidin und Clonidin wirken wahrscheinlich durch die Aktivierung des deszendierenden noradrenergen Systems antinozizeptiv. Sie können innerhalb der ersten $24 \mathrm{~h}$ den Opioidverbrauch und die Schmerzintensität senken.

\section{S-Ketamin \\ $\nabla$}

Intraoperative Gabe Die intraoperative Gabe von S-Ketamin empfiehlt sich insbesondere bei Patienten,

- die bereits eine Opioidtoleranz entwickelt haben,

- die unter neuropathischen Schmerzen leiden,

- bei denen Schmerzen bisher nur schlecht eingestellt sind sowie

- bei Patienten mit einem hohen Risiko, postoperativ starke Schmerzen zu entwickeln [39].

Studienlage Zahlreiche Studien konnten mittlerweile belegen, dass Ketamin die Intensität postoperativer Schmerzen und/oder den postoperativen Opioidverbrauch reduzieren kann [40]. S-Ketamin hat somit auch den Einzug in die 


\section{Management der i.v. S-Ketamingabe}

\begin{tabular}{|c|c|c|}
\hline $\begin{array}{l}0,5 \mathrm{mg} / \mathrm{kg} \text { Bolus } \\
\text { nach Einleitung } \\
\text { vor Schnitt }\end{array}$ & $\begin{array}{l}5-10 \mu \mathrm{g} / \mathrm{kg} / \mathrm{h} \\
\text { über Perfusor } \\
\text { während der OP }\end{array}$ & $\begin{array}{l}\text { Stopp bei } \\
\text { Hautnaht/ } \\
\text { vor Extubation }\end{array}$ \\
\hline
\end{tabular}

Bildnachweis: Carsten Stoetze

\section{Perioperative Gabe von i. v. Lidocain}

\begin{tabular}{|l|l|l|}
\hline $1,5 \mathrm{mg} / \mathrm{kg}$ Bolus \\
nach Einleitung
\end{tabular}$\rightarrow \begin{aligned} & 1,5 \mathrm{mg} / \mathrm{kg} / \mathrm{h} \text { über } \\
& \text { Perfusor, Start } \\
& 30 \mathrm{~min} \text { vor Schnitt }\end{aligned} \rightarrow$\begin{tabular}{l}
$\begin{array}{l}\text { Stopp ca. } 30 \mathrm{~min} \\
\text { nach Hautnaht }\end{array}$ \\
\hline
\end{tabular}

Bildnachweis: Carsten Stoetzer

Abb. 4 (oben)

Abb. 5 (unten)
S3-Leitlinie „Behandlung akuter perioperativer und posttraumatischer Schmerzen" gehalten. Erste Studien weisen zudem darauf hin, dass auch chronische Schmerzpatienten von der perioperativen Ketamingabe profitieren können [41-44].

Off-Label-Use Aus den o.g. Gründen kann daher aktuell die Gabe von S-Ketamin bei Patienten mit zu erwartenden starken Schmerzen nach der Operation und bei chronischen Schmerzpatienten mit großen Operationen erwogen werden, insbesondere wenn ein regionalanästhesiologisches Verfahren nicht möglich ist [41].

- Allerdings handelt es sich hier um einen OffLabel-Use, für den die Patienten präoperativ aufgeklärt werden müssen.

- Abb. 4 zeigt, welches Prozedere laut PogatzkiZahn [41] empfohlen werden kann.

Missbrauch von Ketamin In den letzten Jahren zeichnet sich jedoch in der Drogenszene ein vermehrter Missbrauch von Ketamin ab. Als Droge wird es oft in kristalliner Form, vorzugsweise transnasal, konsumiert. Die Einnahme als Flüssigkeit oder in Form von Tabletten ist selten, da Ketamin hier schnell zu dem wenig halluzinogenen Norketamin umgewandelt wird. In der Szene ist es v.a. wegen seiner medizinisch unerwünschten Nebenwirkungen in der Abklingphase interessant geworden. Es kann zu Halluzinationen mit ausgeprägter Farben- und Formenvielfalt kommen, die mit Gefühlen der Ich-Entgrenzung und Ich-Auflösung verbunden sein können [42]. Höhere Dosierungen können zudem sog. Nahtoderlebnisse zur Folge haben. Aus diesen Gründen kann Ketamin ein gewisses Abhängigkeitspotenzial zugesprochen werden, wenn es als Rauschmittel eingesetzt wird. Ob das jedoch auch einen Einfluss auf die kurze intraoperative Gabe haben könnte, kann bei der derzeitigen Datenlage nicht eindeutig gesagt werden. Es empfiehlt sich jedoch, Ketanest bei Patienten mit bestehender Abhängigkeit vorsichtig und nur kurzzeitig einzusetzen.

\section{Lidocain und Magnesium \\ $\nabla$}

Lidocain Eine stetig steigende Anzahl von Studien kommt zu dem Ergebnis, dass die perioperative Gabe von Lidocain einen positiven Effekt auf das postoperative Schmerzniveau der Patienten zeigt. Besonders untersucht wurden abdominalchirurgische Eingriffe, bei denen keine Epiduralanästhesie etabliert werden konnte (vergleiche hierzu auch die Übersichtsarbeit von Herminghaus et al. [44]). Wir benutzen in unserer Klinik das Lidocain in folgender Dosierung:

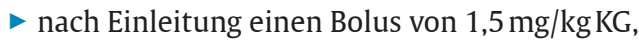

- gefolgt von einer kontinuierlichen Infusion: $1,5 \mathrm{mg} / \mathrm{kg} / \mathrm{h}$; Start etwa $30 \mathrm{~min}$ vor dem Hautschnitt bis $1 \mathrm{~h}$ nach Beendigung der Hautnaht [37].

Allerdings sind auch hier noch weitere Studien notwendig, um den Einfluss von Lidocain auf die Behandlung chronischer Schmerzpatienten besser beurteilen zu können (๑ Abb. 5).

Magnesium Magnesium ist vermutlich durch einen Antagonismus am NMDA-Rezeptor auch an derSchmerzmodulation beteiligt. Eine Übersichtsarbeit von De Oliveira et al. [45] hat 20 Publikationen mit insgesamt 1257 Patienten ausgewertet: Eine perioperative Magnesiumgabe führt sowohl zu einer verringerten Intensität postoperativer Schmerzen als auch zu einem geringeren Opioidbedarf. Allerdings wurden opioidinduzierte Nebenwirkungen wie Übelkeit und Erbrechen nicht reduziert. Nebenwirkungen durch die Gabe von Magnesium hingegen traten nicht auf. Allerdings ist auch hier unklar, inwieweit dieser Effekt auf Patienten mit einer Opioidvorbehandlung übertragbar ist. Insgesamt scheint die perioperative i.v. Magnesiumgabe eine nebenwirkungsarme Möglichkeit in der Reduktion postoperativer Schmerzen zu sein ( $\odot$ Tab. 3).

Fazit Die Behandlung opioidgewöhnter Patienten sowie von Patienten mit manifester (Opioid-) Suchterkrankung impliziert eine Reihe von Besonderheiten. Phänomene wie Toleranz und opioidinduzierte Hyperalgesie spielen dabei oftmals eine ebenso große Rolle wie die generelle Problematik vorbestehender chronischer Schmerzen und deren spezifischer Pathophysiologie. Angepasste Algorithmen (๑ Tab. 3) können beim perioperativen Management dieser Patienten Hilfestellung bieten. Neuere Optionen wie die Gabe von Pregabalin oder $\alpha 2$-Agonisten sind interessante Behandlungsansätze. 


\section{Erweiterter Algorithmus zur Behandlung opioidgewöhnter Patienten}

\section{präoperativ}

\section{Evaluation des präoperativen Status}

- Wirkstoff und Dosis der eingenommenen Substanz

- bei Drogenabusus: Reinheitsgrad der verwendeten Substanz, schwierige Venenverhältnisse?

- Bestehen Erfahrungen mit Unterdosierungen, Entzug?

- Bestehen Begleiterkrankungen (Infektion, kardiovaskuläre Erkrankungen)?

2. Planung des operativen Eingriffs

- frühzeitige interdisziplinäre Kommunikation (Anästhesie, Chirurgie, Akutschmerzdienst)

ggf. Einbeziehung von Bezugspersonen

- bei ambulanten Operationen stationäres Umfeld planen

- Planung des perioperativen Regimes, eines kontinuierlichen Monitorings (Vigilanz, $\mathrm{SpO}_{2}$, EKG), ggfs. postoperativ Überwachung auf der ICU oder IMC

\section{Prämedikation}

- Benzodiazepin (z. B. 7,5 mg Dormicum p. o.)

bei bestehender Opioid- oder Methadonmedikation: übliche Dosierung weiter verabreichen

ggfs. 150-300 mg Pregabalin, $1 \mathrm{~h}$ präoperativ

\section{perioperativ}

\section{Regionalanästhesie}

wenn möglich mit Kathetertechnik

ggfs. Lokalanästhesie, Wundinfiltration

- epiduraler Opioidzusatz nur, wenn kontinuierliches Monitoring der Vitalparameter gewährleistet ist

\section{Allgemeinanästhesie}

- Anästhesie als TIVA oder balanciert möglich, aber 30-100\% höhere Opioiddosen einkalkulieren

- bestehendes transdermales therapeutisches System (TTS) bei kleinen Eingriffen belassen, bei größeren Eingriffen nach Einleitung entfernen, Äquivalenzdosen i.v. verabreichen

- Reminfentanil, Antagonisten (Naloxon o. ä.) und Succinylcholin (Rhabdomyolyse) vermeiden

- bei Vorbehandlung mit Methadon: übliche Dosierung zur gewohnten Zeit beibehalten; zusätzlich operativen Bedarf mit ansetzen (reine $\mu$-Opioidrezeptor-Agonisten)

$\checkmark$ Vorbehandlung mit Partialagonisten (Buprenorphin):

$\triangleright$ in niedriger Dosis zur Analgesie $\rightarrow$ Kombination mit reinen $\mu$-Opioidrezeptor-Agonisten möglich

$\triangleright$ in höherer Dosierung zur Substitution (Tagesdosen von 2-8 mg) $\rightarrow$ präoperative Umstellung auf einen $\mu$-Opioidrezeptor-Agonisten (z. B. Hydromorphon) in äquianalgetischer Dosis. Zusätzlich postoperativen Bedarf anordnen!

- Esketamin, Lidocain oder Dexmedetomidin erwägen

\section{postoperativ}

\section{Analgesie}

- Regionalanästhesie mit Katheter möglichst weiterführen

- in der ersten postoperativen Phase eine patientenkontrollierte i.v. Analgesie (PCIA; z. B. mit Hydromorphon)

- bei Opioiden möglichst Retardpräparate verwenden

- NOPA und Adjuvanzien (Ketamin, Dexmedetomidin, Pregabalin) ausnutzen

- intensive Betreuung des Patienten durch geschultes Personal (Akutschmerzdienst), ggf. psychiatrische Mitbetreuung 


\section{Kernaussagen}

Patienten mit chronischen Schmerzen, die dauerhaft Opioide nehmen, und Patienten, die einen Abusus psychoaktiver Substanzen betreiben, müssen frühzeitig detektiert werden.

Patienten mit aktiver Suchterkrankung sowie mit vorbestehender Opioidtherapie erfordern ein spezielles anästhesiologisches Management.

Falls möglich, empfiehlt es sich, frühzeitig einen Akutschmerzdienst in die Therapieplanung mit einzubeziehen und die Möglichkeit der postoperativen Überwachung der Vitalfunktionen einzuplanen.

Wann immer es Patient und Eingriff zulassen, sollte ein regionalanästhesiologisches Verfahren, bevorzugt in Kathetertechnik, gewählt werden.

Ambulante Operationen können zu einer ungeplanten stationären Aufnahme führen. Es empfiehlt sich daher, diese Patientengruppe geplant in einem stationären Umfeld zu betreuen.

Bei Tachykardie, Hypertension und Schweißausbrüchen muss frühzeitig an einen Opioidentzug gedacht werden.

Entzugssymptome durch restriktive Opioidgabe erhöhen die Rückfallgefahr mehr als eine adäquate, bedarfsorientierte Gabe von Opioiden.

Antagonisten wie Flumazenil, Naloxon und Neostigmin sollten ebenso wie das kurzwirksame Opioid Remifentanil nicht eingesetzt werden.

Weitere Therapieansätze wie die Gabe von S-Ketamin, Pregabalin, Lidocain und Magnesium sind ein vielversprechender Ansatz in der Behandlung opioidgewöhnter Patienten.
Interessenkonflikt Die Autoren erklären, dass keine Interessenkonflikte vorliegen.

Beitrag online zu finden unter http://dx.doi. org/10.1055/s-0041-100389

\section{VNR 2760512015147120394}

\section{Literaturverzeichnis}

1 Bericht zum Substitutionsregister, BfArM, 2014

2 Kraus L, Pabst A, Gomes de Matos E, Piontek D. Kurzbericht Emidemiologischer Suchtsurvey 2012. Tabellenband: Prävalenz der Medikamenteneinnahme und medikamentenbezogener Störungen nach Geschlecht und Alter im Jahr 2012. 2013. Online verfügbar unter http://www.ift.de/index.php?id=429

3 Kraus L, Pabst A, Gomes de Matos E, Piontek D. Kurzbericht Emidemiologischer Suchtsurvey 2012. Tabellenband: Prävalenz des Konsums illegaler Drogen, multipler Drogenerfahrung und drogenbezogener Störungen nach Geschlecht und Alter im Jahr 2012. 2014. Online verfügbar unter http://www.ift.de/index.php?id=429

4 Kraus L, Pabst A, Gomes de Matos E, Piontek D. Kurzbericht Emidemiologischer Suchtsurvey 2012. Tabellenband: Prävalenz des Alkoholkonsums, episodischen Rauschtrinkens und alkoholbezogener Störungen nach Geschlecht und Alter im Jahr 2012. 2014. Online verfügbar unter http://www.ift.de/index.php?id=429

5 Karst M. Chronic pain - an update. Anästh Intensivmed 2014; 55: 190-197

$6 \mathrm{Kim}$ SH, Stoicea N, Soghomonyan S, Bergese SD. Intraoperative use of remifentanil and opioid induced hyperalgesia/acute opioid tolerance: systematic review. Front Pharmacol 2014; 5: 108

7 Chu LF, Clark DJ, Angst MS. Opioid tolerance and hyperalgesia in chronic pain patients after one month of oral morphine therapy: a preliminary prospective study. J Pain 2006; 7: 43-48

8 Ballantyne JC, Mao J. Opioid therapy for chronic pain. N Engl J Med 2003; 349: 1943-1953

9 Williams JT, Ingram SL, Henderson G et al. Regulation of $\mu$-opioid receptors: desensitization, phosphorylation, internalization, and tolerance. Pharmacol Rev 2013; 65: 223-254

10 Stromer W, Michaeli K, Sandner-Kiesling A. Perioperative pain therapy in opioid abuse. Eur J Anaesthesiol 2013; 30 $55-64$

11 Raffa RB, Pergolizzi Jr. JV. Opioid-induced hyperalgesia: is it clinically relevant for the treatment of pain patients? Pain Manag Nurs 2013; 14: e67-83

12 Carroll IR, Angst MS, ClarkJD. Management of perioperative pain in patients chronically consuming opioids. Reg Anesth Pain Med 2004; 29: 576-591

\section{Literatur online}

Dr. med. Jörg Filitz, MA, ist Oberarzt an der Klinik für Anästhesiologie und Intensivmedizin der Medizinischen Hochschule Hannover (MHH) und zuständig für den

Akutschmerzdienst. E-Mail: filitz.joerg@mh-hannover.de
Das vollständige Literaturverzeichnis zu diesem Beitrag finden Sie im Internet:

Abonnenten und Nichtabonnenten können unte „www.thieme-connect.de/ejournals“ die Seite der AINS aufrufen und beim jeweiligen Artikel auf „Zusatzmaterial“ klicken - hier ist die Literatur für alle frei zugänglich.

Abonnenten können alternativ über ihren persönlichen Zugang an das Literaturverzeichnis gelangen. Wie das funktioniert, lesen Sie unter: http://www.thiemeconnect.de/ejournals/help\#SoRegistrieren 


\section{CME-Fragen Opioidgewöhnte Patienten}

Welche Aussage über die Behandlungsansätze opioidgewöhnter Patienten trifft zu?

A Patienten mit ehemaligem Opioidabusus, die aktuell stabil substituiert werden, benötigen häufig höhere Opioiddosen. Diese sollten schon in der Prämedikation verordnet werden.

B Patienten die erfolgreich entwöhnt wurden, dürfen wegen der erhöhten Rückfallgefahr postoperativ keine Opioide bekommen.

C Es gibt verschiedene Gruppen von Patienten mit Opioidgewöhnung, deren Behandlungsansatz jedoch identisch ist.

D In der postoperativen Schmerztherapie sollen vorzugsweise Opioide mit unretardierter Galenik Verwendung finden.

E Patienten mit vorbestehender Opioidtherapie erfordern ein spezielles anästhesiologisches Management.

\section{Welche Aussage ist falsch?}

A Patienten, die einen aktiven Substanzmissbrauch betreiben, können postoperativ über vermehrte Schmerzen klagen.

B Aufgrund der komplexen Wechselwirkungen zwischen psychischen und organischen Einflüssen empfiehlt es sich, nicht ambulant zu behandeln.

c Durch die perioperative Gabe von Magnesium kann die Intensität postoperativer Schmerzen sowie das Auftreten opioidbedingter Nebenwirkungen (Übelkeit, Erbrechen) reduziert werden.

D Wann immer möglich, sollte bei opioidgewöhnten Patienten ein regionalanästhesiologisches Verfahren durchgeführt werden.

E Die perioperative Analgesie opioidgewöhnter Patienten setzt sich aus Basismedikation und Bedarf der Operation zusammen.

Was sollte bei Vorbehandlung mit einem hochdosierten Buprenorphin-Pflaster auf jeden Fall beachtet werden?

A Das Pflaster sollte präoperativ immer entfernt werden.

B Buprenorphin bewirkt als Antagonist des k-Opioidrezeptors eine verminderte Wirkung reiner $\mu$-Opioidrezeptor-Agonisten.

c Als Partialagonist des $\mu$-Opioidrezeptors hat Buprenorphin nur eine schwache analgetische Potenz.

D Die transdermale Aufnahme von Buprenorphin kann durch Flüssigkeitsverschiebungen und Temperaturschwankungen stark beeinflusst werden.

E Buprenorphin hat eine geringe Affinität zum $\mu$-Opioidrezeptor und verursacht damit keine Atemdepression.

Welche Aussage ist richtig?

A Unter dem Begriff der Toleranz versteht man eine Linksverschiebung der Dosis-Wirkungs-Kurve.

B Toleranz und opioidinduzierte Hyperalgesie sind das Gleiche.

C Die opioidinduzierte Hyperalgesie ist eher akademisch und spielt nur eine untergeordnete Rolle.

D Bei der Toleranz wird - anders als bei der opioidinduzierten Hyperalgesie - die Schmerzempfindungsschwelle nicht herabgesetzt.

E Sind postoperative Schmerzen durch eine opioidinduzierte Hyperalgesie bedingt, müssen sie durch höhere Opioidgaben therapiert werden.

5 Welche Aussage trifft für den Einsatz von Dexmedetomidin in der perioperativen Schmerztherapie zu?

A Es zeigt gegenüber Clonidin mehr kardiovaskuläre Nebenwirkungen.

B Für Dexmedetomidin konnte ein opioidsparender Effekt bei Patienten mit aktiver Sucht eindeutig nachgewiesen werden.

c Dexmedetomidin bewirkt eine relevante Zunahme postoperative Übelkeit.

D Clonidin ist als der potentere Agonist zentraler $\alpha 2$-Rezeptoren grundsätzlich stärker analgetisch wirksam.

E Die analgetische Wirkung von Dexmedetomidin erklärt sich durch eine Aktivierung des deszendierenden noradrenergen Systems.
Was ist bei Patienten mit einer Vorbehandlung mit starken Opioiden obsolet?

A regionalanästhesiologische Verfahren ohne zusätzliche Gaben von Opioiden

B Vollnarkosen mit Remifentanil als einzigem Opioid

C Prämedikation mit Pregabalin oder Gabapentin

D Bevorzugung einer stationären Aufnahme trotz der Möglichkeit einer ambulanten Behandlung

E Beibehaltung und Fortsetzung einer Einnahme eines Partialagonisten im Rahmen der perioperativen Schmerztherapie

7 Welche Aussage zu S-Ketamin trifft zu?

A S-Ketamin ist aufgrund des günstigen Nebenwirkungspotenzials das ideale Analgetikum bei opioidgewöhnten Patienten.

B Bei Patienten mit positiver Suchtanamnese sollte es wegen der Gefahr induzierter Albträume keine Verwendung finden.

C Bisher gibt es keine Untersuchungen, die einen positiven Effekt von S-Ketamin in der Schmerztherapie zeigen.

D S-Ketamin kann die Intensität postoperativer Schmerzen und den Opioidverbrauch senken.

E S-Ketamin zeigt nur in Kombination mit Regionalanästhesie gute Ergebnisse bei der postoperativen Analgesie.

8

Was trifft nicht auf das Management opioidgewöhnter Patienten zu?

Opioidbehandlung und deren Begleitumstände können zu Episoden erheblicher Schmerzverstärkung führen.

B Vorbestehende chronische Schmerzen/deren Therapie müssen bei der Planung therapeutischer Interventionen berücksichtigt werden.

c Auswahl, Kombination, Dosierung, Anwendungsart und Anwendungsdauer geeigneter medikamentöser und nicht-medikamentöser Verfahren sind für das Outcome entscheidend.

D Die Verwendung von Opioiden unterstützt aufgrund der pronozizeptiven Eigenschaften das Fortschreiten von Sensibilisierungsprozessen und sollten daher konsequent vermieden werden.

E Eine differenzierte Anamnese kann opioidgewöhnte Patienten frühzeitig erkennen und erleichtert das perioperative Management.

9 Welche Aussage zu Patienten unter Substitutionstherapie und chronischen Schmerzpatienten trifft zu? Levomethadon und Methadonracemat können 1:1 ersetzt werden. Das geeignete Narkoseverfahren für kurze Eingriffe ist eine Allgemeinanästhesie mit Propofol und Ultiva.

C Sie geben am Ende der Operation Naloxon, um ein rasches Erwachen zu erreichen.

D Die Aufwachphase sollte langsam und stressfrei in einer ruhigen Umgebung erfolgen.

E Die sublingual-transmukosalen Gabe von Buprenorphin bei Vorbehandlung mit reinen $\mu$-Agonisten führt im Regelfall zu einer Wirkabschwächung des Opioides mit Zunahme der Schmerzen.

Welche der Aussagen zu anamnestischen und therapeutischen Ansätzen in der perioperativen Betreuung opioidgewöhnter Patienten ist nicht zutreffend?

A In der Anamnese sollte differenziert nach der Menge und der Einnahmehäufigkeit der verwendeten Substanzen gefragt werden.

B Clonidin kann als Adjuvanz den postoperativen Verlauf günstig beeinflussen

C Ein Entzugssyndrom kann prinzipiell auch während einer Allgemeinanästhesie auftreten.

D Der perioperative Opioidbedarf opioidgewöhnter Patienten ist meist geringer als bei opioidnaiven Patienten

E Das Phänomen der Toleranz entwickelt sich v.a. unter den ZNS-dämpfenden, jedoch kaum unter den ZNS-aktivierenden Substanzen. 